Original Article

\title{
A Survey to Assess the Number, Shape and Attachment of the Freni in the Maxillary and Mandibular Arches in South Indian Population
}

\section{Sorte Nandita ${ }^{1}$, Vinaya Bhat ${ }^{2}$, Chethan Hegde ${ }^{3}$}

${ }^{1}$ Post Graduate student, ${ }^{2}$ Professor, ${ }^{3}$ Professor and HOD , Department of Prosthodontics, A. B. Shetty M emorial Institute of Dental Sciences, NITTE (Deemed to be University), M angalore.

Corresponding Author : Vinaya Bhat, Professor, Department of Prosthodontics, A.B. Shetty M emorial Institute of Dental Sciences, NITTE (Deemed to be University), M angalore. M obile : +919481921180 E-mail : drvinayabhat@gmail.com

Received

: 30.01 .2017

Review Completed : 03.05.2017

Accepted

: 14.04 .2018

Keywords : labial frenum, buccal frnum, lingual frenum

\begin{tabular}{|c|}
\hline Access this article online \\
\hline Quick Response Code \\
\hline
\end{tabular}

\section{Abstract}

Frenum is one of the most variable anatomical structures present in the oral cavity which can be defined as a "fibrous band of tissue attached to the bone of the mandible and maxillae, and is present superficial to muscle attachments." ${ }^{11}$ In the present study, hundred individuals aged 18 to 40 years were selected randomly. The patients were examined (i) To assess the number, shape and attachment of the freni in the maxillary arch and (ii) To assess the number, shape and attachment of the freni in the mandibular arch in south Indian population. It was concluded that single labial frenum was more common in both the arches, buccal frenum in maxilla were multiple. Whereas, in mandible buccal frenum had single frenal attachment. V-shaped frenal attachment was more in the maxillary and mandibular arches exception being the lingual frenum which showed prevalence pertaining to fan shape.

\section{Introduction}

Frenum is one of the most variable anatomical structures present in the oral cavity which can be defined as a "fibrous band of tissue attached to the bone of the mandible and maxillae, and is presenting superficial to muscle attachments." ${ }^{1}$ (Fig. 1)

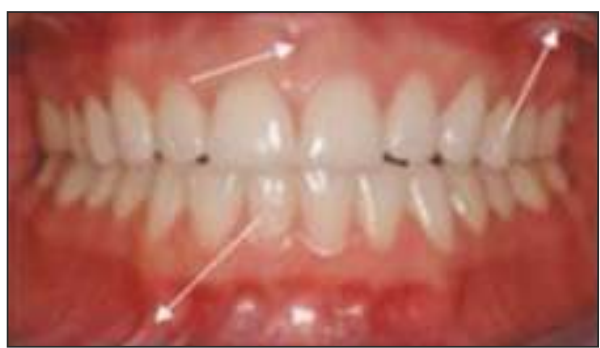

Fig. 1 ; Frenal attachment

Freni are mostly seen in the vestibular mucosa of the mandible and maxilla, usually in the midline or premolar region. The maxillary/mandibular labial frena and the lingual frenum are the most notable frena of the normal oral cavity. ${ }^{2}$ among all the other frena, maxillary frena are dynamic structures that are subjected to variations during different stages of human growth and development.

Histologically, it is made up of loose fibrous connective tissue, abundance of elastic fibers along with a few striated muscle fibers that arise from the muscle bundles of the lip on either side of the midline; however, the controversy regarding the histology of frenum still remains. ${ }^{4}$ The primary function is to provide stability to the upper lip, lower lip, and tongue. ${ }^{2}$

The maxillary labial frenum has been implicated in the development of central incisor diastemas, and the lingual mandibular is deemed responsible for most cases of ankyloglossia. The lingual frenulum, a small fold of mucous membrane that connects the middle of the sublingual face of the tongue to the floor of the mouth, interferes in the tongue movements and itsfunctions. 
The frenum also plays a very important role in the fabrication of dentures. It provides retention and stability to the dentures. If the freni notches in complete dentures are not relieved properly, it can cause trauma to the freni and also there will be improper seating of the denture base. High frenum attachment in maxilla or mandible due to stress concentration will lead to fracture of the dentures. ${ }^{8}$

Various types of frenal attachments have been documented in the literature such as mucosal, gingival, papillary and papillary penetrating as proposed by Mirko et al ${ }^{9}$. Later Sewerin ${ }^{7}$ further classified the variations of frenum as normal frenum, normal frenum with a nodule, normal frenum with appendix, normal frenum with nichum, bifid labial frenum, persistent tectolabial frenum, double frenum, and wider frenum.

However, very few studies have been documented that were carried out in children pertaining to shape and attachment. ${ }^{6}$ No studies have been done pertaining to the number of frenal attachment and the shape and attachment in adults of South India. Hence, this study was taken up to find out the prevalence of frenal variations in a diverse ethnic population in South India.

\section{Materials and methods}

Sample selection: Hundred individuals aged 18 to 40 years were selected randomly from the Department of Prosthodontics including crown and bridge, A. B. Shetty Memorial Institute of Dental Sciences, Mangalore. Individuals who had undergone surgical correction of the frenum were excluded from this study.

\section{Methodology}

The patients were then examined using diagnostic instruments and the findings recorded.

The following aspects were examined in each subject in the maxilla and mandible in each

Subject:

1. The type of the frenum attachment as given in the classification by $\mathrm{M}$ irko ${ }^{9}$
2. The number of each freni.

3. The shape of each freni

\section{Results}

1. Type of mucosal frenal attachment in maxillary arch (labial) were seen to be $70 / 100$ and $30 / 100$ were gingival (fig. 2)

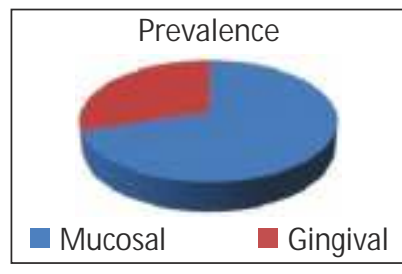

2. Type of mucosal frenal attachment in maxillary arch (right buccal) were seen to be $63 / 100$ and $37 / 100$ were gingival (fig. 3)

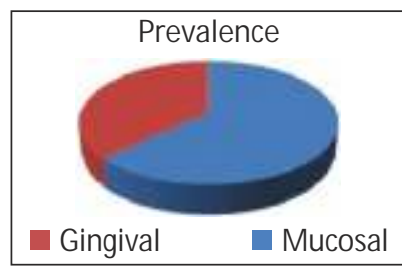

3. Type of mucosal frenal attachment in maxillary arch (left buccal) were seen to be $61 / 100$ and $39 / 100$ were gingival (fig. 4)

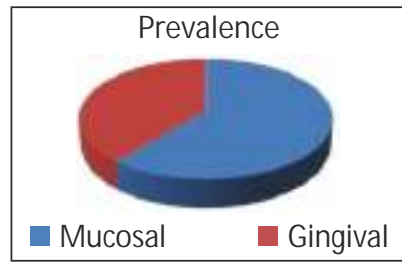

4. Type of mucosal frenal attachment in mandibular arch (labial) were seen to be $76 / 100$ and $24 / 100$ were gingival (fig. 5)

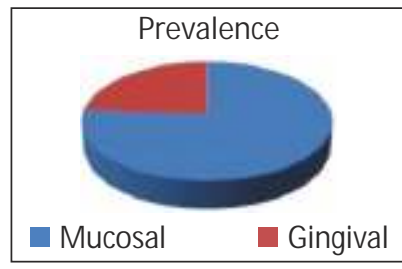

5. Type of mucosal frenal attachment in mandibular arch (right buccal) were seen to be $79 / 100$ and $21 / 100$ were gingival (fig. 6)

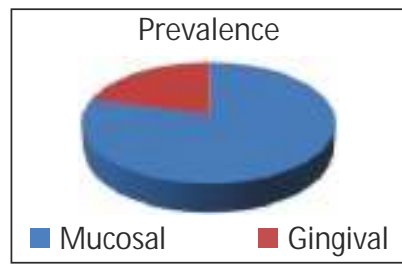

6. Type of mucosal frenal attachment in mandibular arch (left buccal) were seen to be $73 / 100$ and $27 / 100$ were gingival (fig. 6)
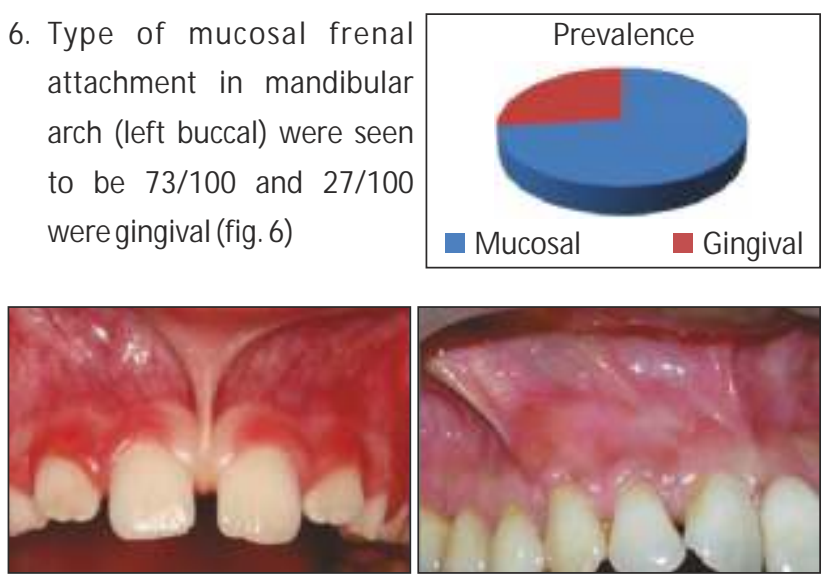

Fig. $\mathbf{2}$ : Labial frenum

Fig. 3 : M axillary right buccal frenum 

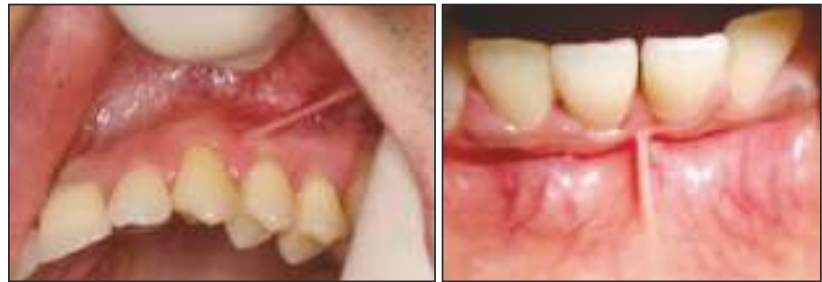

Fig. 4 : Maxillary left buccal frenum

Fig. 5 : M andibular labial fenum

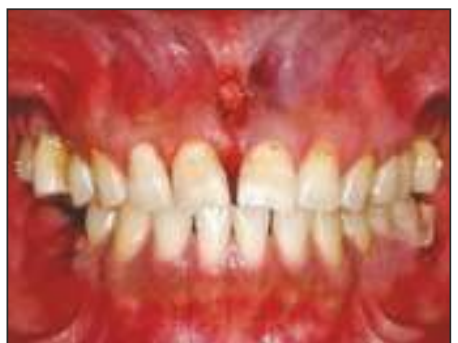

Fig. 6 : M andibular right and left buccal frenum

7. Number of patients having in the maxillary arch were $92 / 100$ and those having more than one were $8 / 100$ (fig. 7)

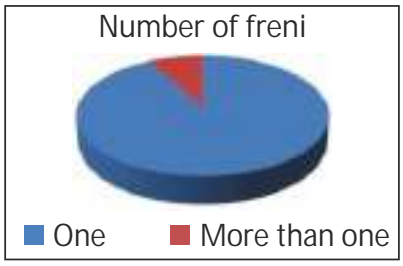

8. Number of patients having one right buccal frenum in the maxillary arch were $36 / 100$ and those having more than one were 64/ 100 (fig. 8)

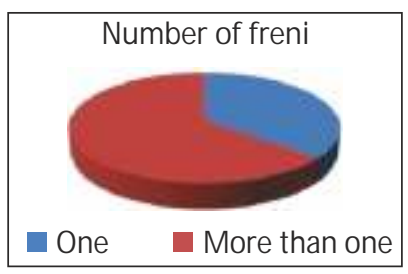

9. Number of patients having one left buccal frenum in the maxillary arch were 32/100 and those having more than onewere 68/100 (fig. 9)

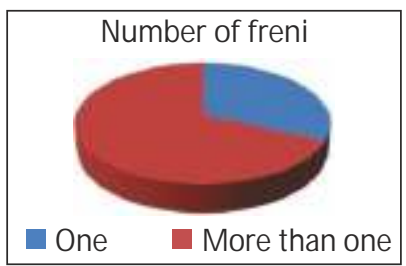

10.Number of patients having one right buccal frenum in the mandibular arch were 87/100 and those having more than one were 13/100 (fig. 10)

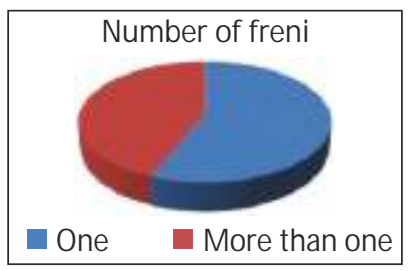

11.Number of patients having one left buccal frenum in the mandibular arch were 82/ 100 and those having more than onewere 18/100 (fig. 10)

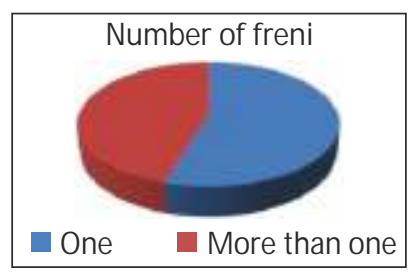

12.Number of patients having one labial frenum in the mandibular arch were 73/100 and those having more than one were $27 / 100$ (fig. 11)

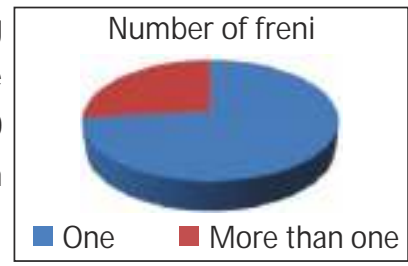

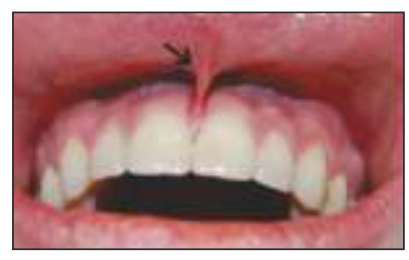

Fig. 7 : Single maxillary labial frenum

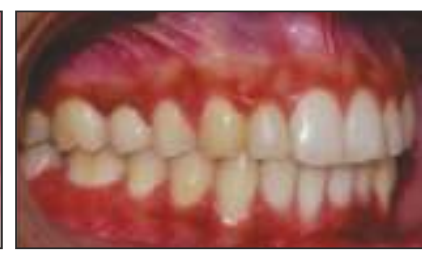

Fig. 8 : More than one maxillary right buccal frenum

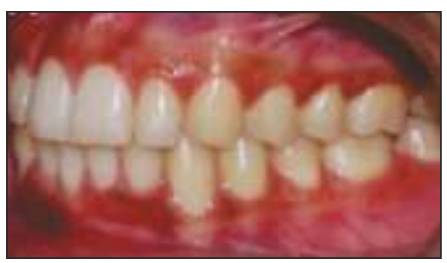

Fig. 9 : M ore than one maxillary left buccal frenum

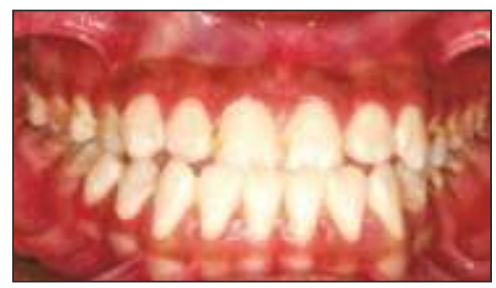

Fig.10 : M ore than one left and right mandibular buccal freni

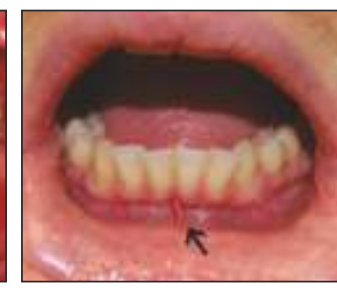

Fig. 11 : Single mandiblar labial frenum
13.Type of $V$-shaped frenal attachment in the maxillary arch (labial) were 87/100 and 13/100 were fan shaped (fig. 12)

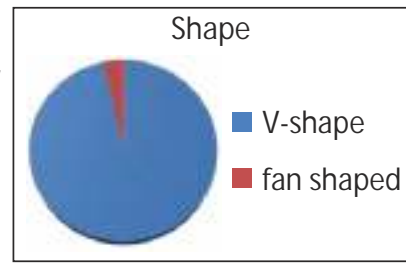

14.Type of V-shaped frenal attachment in the maxillary arch (right buccal) were $89 / 100$ and $11 / 100$ were fan shaped (fig. 13)

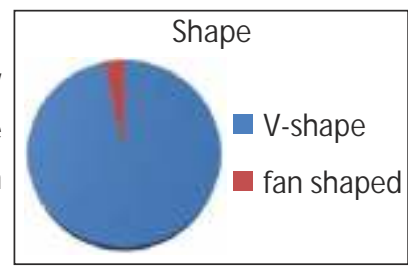

15.Type of V-shaped frenal attachment in the maxillary arch (left buccal) were $89 / 100$ and $11 / 100$ were fan shaped (fig. 14)

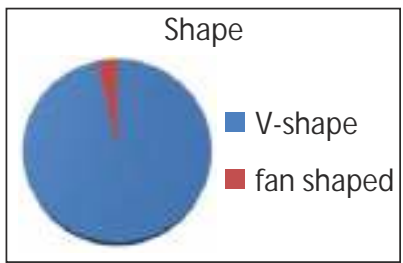




16.Type of V-shaped frenal
atta chment in th e
mandibular arch (labial) were
$72 / 100$ and $28 / 100$ were fan
shaped (fig. 15)

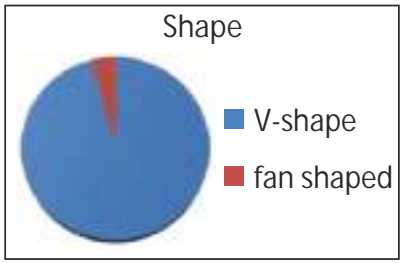

17.Type of V-shaped frenal attachment in the mandibular arch (right buccal) were $86 / 100$ and $14 / 100$ were fan shaped (fig. 16)

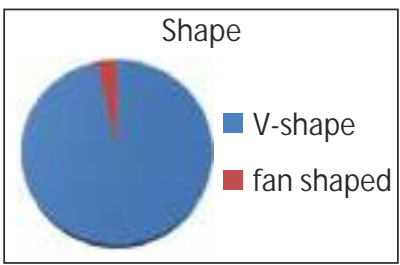

18.Type of V-shaped frenal attachment in the mandibular arch (left buccal) were $91 / 100$ and $9 / 100$ were fan shaped (fig. 16)

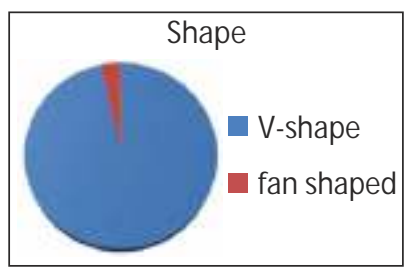

19.Type of V-shaped frenal attachment in the mandibular arch (lingual) were $43 / 100$ and $57 / 100$ were fan shaped (fig. 17)
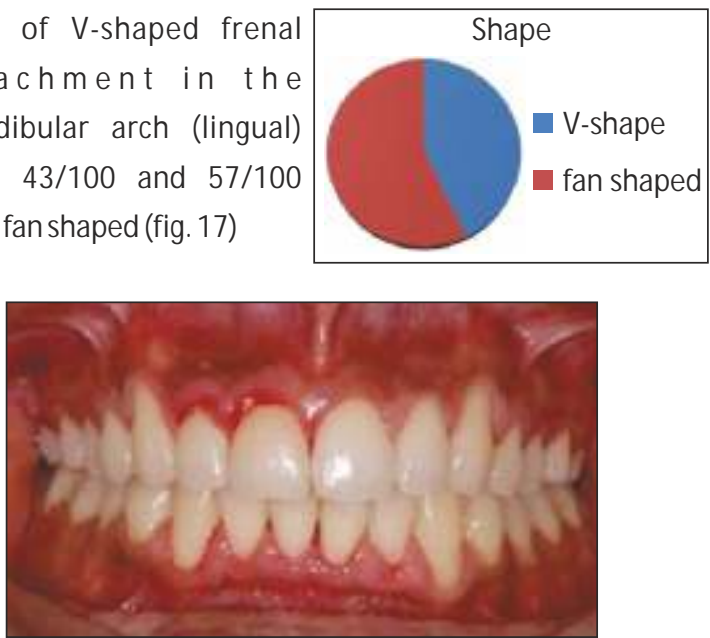

Fig. 12 V-shape maxillary labial frenum

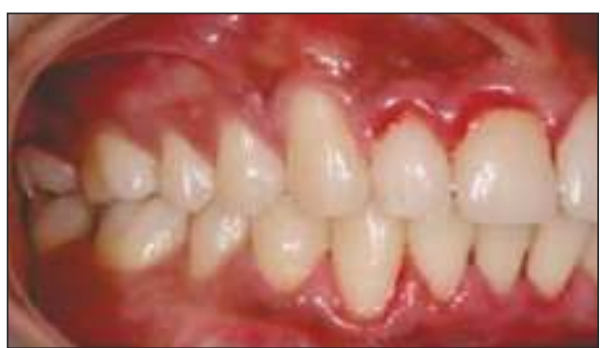

Fig. 13 V-shape maxillary right buccal frenum

\section{Discussion}

The maxillary labial frenum has been the cause for development of central incisor diastemas, and the lingual frenum is responsible for most cases of ankyloglossia. The frenum also plays a very important role in the fabrication of



Fig. 14 V-shape maxillary left buccal frenum

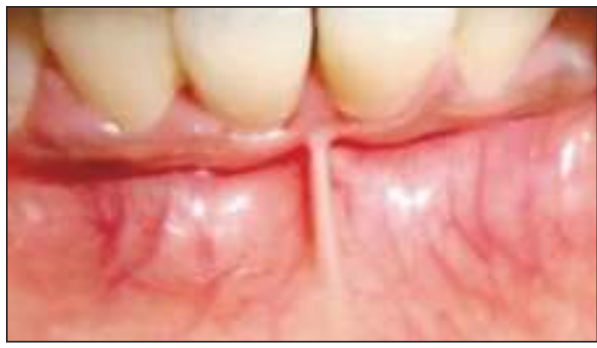

Fig. 15 V- shape mandibular labial frenum

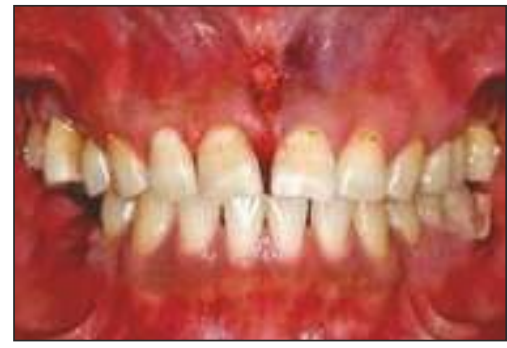

Fig. $16 \mathrm{~V}$-shape mandibular right and left freni

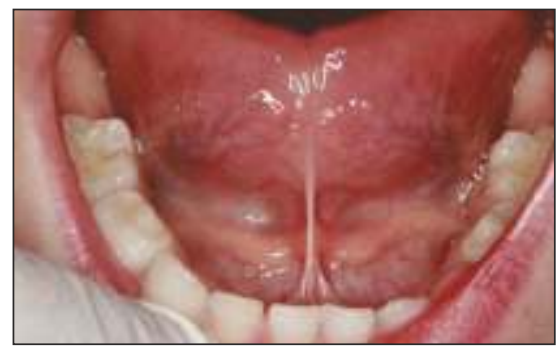

Fig. 17 Fan shape lingual frenum

dentures in providing retention and stability to the dentures. If the freni notches in complete dentures are not relieved properly, it can cause trauma to the freni and also there will be improper seating of the denture base. If a large

frenal notch is made this can lead to areas of stress concentration leading to fracture of denture. ${ }^{8}$

Various classifications have been proposed regarding the variations in frenum, but the most widely accepted classifications were given by Mirko et al., ${ }^{9}$ and Sewerin. ${ }^{7}$ 
Depending upon the extension of attachment of fibers, frena have been classified as: ${ }^{9}$

1. Mucosal - when the frenal fibers are attached up to muco-gingival junction

2. Gingival - when fibers are inserted within attached gingival

3. Papillary - When fibers are extending into inter dental papilla

4. Mucosal - when the frenal fibers are attached up to muco-gingivaljunction

5. Gingival - when fibers are inserted within attached gingival

6. Papillary - When fibers are extending into inter dental papilla

7. Papilla penetrating - When the frenal fibers cross the alveolar process and extend up to palatine papilla.

Sewerin ${ }^{7}$ has also classified the variations of frenum as:

- Normal frenum

- Normal frenum with a nodule

- Normal frenum with appendix

- Normal frenum with nichum

- Bifid labial frenum

- Persistent tectolabial frenum

- Double frenum

- Wider frenum.

In a study conducted by Priyanka M, Sruthi Ret al., stated that frenal attachments are thin folds of mucous membrane with enclosed muscle fibers that attach the lips to the alveolar mucosa and underlying periosteum. Most often, during the oral examination of the patient the dentist gives very little importance to the frenum, for assessing its morphology and attachment. However, it has been seen that an abnormal frenum can be an indicator of a syndrome. This paper highlighted the different frenal attachments seen in association with various syndromic as well as non-syndromic conditions. ${ }^{2}$

S Linda Christabel et al., conducted a cross-sectional study to examine the prevalence of the various types of maxillary frenal attachments and its morphology in children. Total number of children who were examined was 931. They found that the most prevalent type of frenal attachment among all is gingival type followed by mucosal type of attachment. $49.5 \%$ were gingival type, $38.8 \%$ were mucosal type, $9.8 \%$ were papillary type and $1.9 \%$ were papillary penetrating type .The prevalence type had no gender difference but the age had significant association. The occurrence of papillary penetrating type decreases with age. The most prevalent morphological type is simple frenum $97 \%$ followed by simple frenum with nodule which is $2.6 \%$ and bifid labial frenum which is $0.4 \%$. The conclusion drawn was the prevalent type among Chennai children is gingival type. The papillary penetrating type of frenal attachment decreases with age. ${ }^{6}$

According to the classification by Mirko et al., in the maxillary arch $70 \%$ of the patients presented with mucosal type of labial frenal attachment while in $30 \%$ of the patients, gingival type was seen. The right buccal frenal attachment showed $63 \%$ as mucosal and the remaining $37 \%$ as gingival whereas, in the left buccal it was $61 \%$ mucosal and $39 \%$ gingival. In the mandibular arch the labial frenal attachment was $76 \%$ mucosal and $24 \%$ gingival. The right buccal showed $79 \%$ mucosal and $21 \%$ gingival; left showed it was seen to be $73 \%$ buccal and $27 \%$ gingival.

The numbers of patients having one labial frenum in the maxillary arch were $92 \%$ and the remaining $8 \%$ had more than one frenum. One right buccal frenum was seen in $36 \%$ of the population and the rest which were $64 \%$ had more than one. The left buccal frenum showed $32 \%$ of the population having one frenum and $68 \%$ having more than one. Whereas, in the mandibular arch the statistics showed $73 \%$ having one labial frenum and $27 \%$ having more than one. $87 \%$ showed one right buccal frenum showed one compared to $13 \%$ showing more than one. In the left buccal frenum $82 \%$ showed one frenum and $18 \%$ showed more than one.

Shape of the maxillary labial frenum was found to be Vshaped in $87 \%$ of the population and fan shaped in $13 \%$ of the population. The left and right buccal showed $89 \%$ of $\mathrm{V}$ shaped and $11 \%$ of fan shaped. In the mandibular arch $72 \%$ 
of labial frenum showed V-shaped and the rest $28 \%$ were fan shaped. The right buccal had a value of $86 \%$ for $V$ shaped and $14 \%$ as fan shaped. The left buccal were $91 \% \mathrm{~V}$ shaped and $9 \%$ fan shaped. The lingual frenal attachment showed $43 \%$ V-shaped and $57 \%$ fan shaped.

\section{Conclusion}

The study was done in patients of age group $18-40$ years as a whole. The numbers of people having a single labial

\section{References}

1. Zarb GA, Bryant SR. Preprosthetic surgery: Improving the patients denture-bearing areas and ridge relations. In: Zarb GA, Bolender $\mathrm{CL}$, editors. Prosthodontic Treatment for Edentulous Patients, Complete Dentures and Implant-Supported Prostheses. 12th ed. Amsterdam: Elsevier; 2004. p. 103.

2. Priyanka M, Sruthi R, Ramakrishnan T, Emmadi P, Ambalavanan N. An overview of frenal attachments. J Indian Soc Periodontol 2013;17:12-5.

3. Delli K, Livas C, Sculean A, Katsaros C, Bornstein M M . Facts and myths regarding the maxillary midline frenum and its treatment: $A$ systematic review of the literature. Quintessence Int 2013;44:177-87.

4. Henry SW, Levin MP, Tsaknis PJ. Histologic features of the superior labial frenum.J Periodontol 1976;47:25-8.

5. Irene Queiroz Marchesan; Roberta Lopes de Castro Martinelli, Reinaldo Jordão Gusmão. Lingual frenulum: changes after frenectomy. frenum in the maxillary and mandibular arches were more than those having multiple. Whereas, the buccal freni in the maxilla showed more than one attachment. The mandibular arch had more of single frenal attachment buccally on both sides. V-shaped frenal attachment was more prevalent in the maxillary and mandibular arches exception being the lingual frenum which had more statistical data pertaining to fan shaped.

J. Soc. Bras. Fonoaudiol. vol.24 no.4 São Paulo 2012

6. S Linda Christabel, Deepa Gurunathan. Prevalence of Type of Frenal Attachment and Morphology of Frenum in Children, Chennai, Tamil Nadu. World Journal of Dentistry, October-December 2015;6(4):203207

7. Sewerin I. Prevalence of variations and anomalies of the upper labial frenum. Acta Odontol Scand 1971;29:487-96.

8. El-Sheikh, Al-Zahrani. Causes of denture fracture: A survey. Saudi Dental Journal, Volume 18, No. 3, September - December 2006:18(3):149-154

9. Mirko $P$, Miroslav $S$, Lubor M. Significance of the labial frenum attachment in periodontal disease in man. Part I. Classification and epidemiology of the labial frenum attachment. J Periodontol 1974;45:891-4. 